\title{
Antineutrophil Cytoplasmic Antibody-Associated Vasculitis in Korea: A Narrative Review
}

\author{
Chan-Bum Choi ${ }^{1}$, Yong-Beom Park ${ }^{2}$, and Sang-Won Lee ${ }^{2}$ \\ ${ }^{1}$ Department of Rheumatology, Hanyang University Hospital for Rheumatic Diseases, Seoul; \\ ${ }^{2}$ Division of Rheumatology, Department of Internal Medicine, and Institute for Immunology and Immunological Diseases, Yonsei University \\ College of Medicine, Seoul, Korea.
}

\begin{abstract}
Antineutrophil cytoplasmic antibody-associated vasculitis (AAV) is a group of systemic necrotising vasculitides, which often involve small vessels, and which lead to few or no immune deposits in affected organs. According to clinical manifestations and pathological features, AAV is classified into three variants: microscopic polyangiitis, granulomatosis with polyangiitis (GPA), and eosinophilic GPA. The American College of Rheumatology 1990 criteria contributed to the classification of AAV, although currently the algorithm suggested by the European Medicines Agency in 2007 and the Chapel Hill Consensus Conference Nomenclature of Vasculitides proposed in 2012 have encouraged physicians to classify AAV patients properly. So far, there have been noticeable advancements in studies on the pathophysiology of AAV and the classification criteria for AAV in Western countries. However, studies analysing clinical features of Korean patients with AAV have only been conducted and reported since 2000. One year-, 5 year-, and 10 year-cumulative patient survival rates are reported as $96.1,94.8$, and $92.8 \%$. Furthermore, initial vasculitis activity, prognostic factor score, age and specific organ-involvement have been found to be associated with either all-cause mortality or poor disease course. The rate of serious infection is $28.6 \%$, and 1 year-, 5 year- and 10 year-cumulative hospitalised infection free survival rates range from $85.1 \%$ to $72.7 \%$. The overall standardised incidence ratio of cancer in AAV patients was deemed 1.43 compared to the general Korean population.
\end{abstract}

Key Words: Antineutrophil cytoplasmic antibody, vasculitis, Korea

\section{INTRODUCTION}

Antineutrophil cytoplasmic antibody (ANCA)-associated vasculitis (AAV) is a group of systemic necrotising vasculitides, which often involve small vessels, and which lead to few or no immune deposits in affected organs. ${ }^{1}$ According to clinical manifestations and pathological features, AAV is classified into three variants: microscopic polyangiitis (MPA), granulo-

\footnotetext{
Received: May 23, 2018

Corresponding author: Sang-Won Lee, MD, PhD, Division of Rheumatology, Department of Internal Medicine, and Institute for Immunology and Immunological Diseases, Yonsei University College of Medicine, 50-1 Yonsei-ro, Seodaemun-gu, Seoul 03722, Korea.

Tel: 82-2-2228-1987, Fax: 82-2-393-6884, E-mail: sangwonlee@yuhs.ac

-The authors have no potential conflicts of interest to disclose.

(C) Copyright: Yonsei University College of Medicine 2019

This is an Open Access article distributed under the terms of the Creative Commons Attribution Non-Commercial License (https://creativecommons.org/licenses/ by-nc/4.0) which permits unrestricted non-commercial use, distribution, and reproduction in any medium, provided the original work is properly cited.
}

matosis with polyangiitis (GPA), and eosinophilic GPA (EGPA). ${ }^{2}$ GPA and EGPA are identical to what have been called Wegener's granulomatosis and Churg-Strauss syndrome, although GPA and EGPA are more often and widely used. ${ }^{1}$ MPA mainly induces rapid progressive necrotising glomerulonephritis, and it occasionally provokes pulmonary capillaritis or alveolar haemorrhage. ${ }^{1,2}$ GPA often involves the upper and lower respiratory tracts, and it also affects the kidneys, leading to necrotising glomerulonephritis. EGPA is commonly accompanied by allergic features such as asthma and eosinophilia, and it frequently involves the lungs and skin. ${ }^{3,4}$

Only a few original articles and one review article have reported clinical features and prognosis in Korean patients with AAV since $2000{ }^{5,6}$ In this review, we searched articles, not case reports, with titles and abstracts including "ANCA," "vasculitis," "microscopic polyangiitis," "granulomatosis with polyangiitis (GPA; Wegener's granulomatosis)," "eosinophilic granulomatosis with polyangiitis (EGPA; Churg-Strauss syndrome)," and "KOREA" in PubMed and analysed them. We primarily 
focused on reclassifying according to new criteria and clinical and epidemiological aspects of AAV in the present review. In addition, we provided relevant indices through which to estimate the current activity and to predict outcomes in Korean patients with AAV.

\section{A GLANCE AT THE PATHOGENSIS OF AAV}

Antineutrophil cytoplasmic antibodies recognise typical antigens in the cytoplasm of neutrophils, myeloperoxidase (MPO) and proteinase 3 (PR3). ${ }^{7}$ In healthy individuals, ANCAs exhibit beneficial homeostatic functions, functioning as "natural ANCAs" or "non-pathogenic ANCAs." Natural ANCAs have lower titres, lower avidity, less subclass diversity, and less capability to activate neutrophil than pathogenic ANCAs. ${ }^{8}$ However, when this regulation is broken, ANCAs initiate pathogenic autoimmunity. Endogenous and exogenous stimuli can convert natural ANCAs to pathogenic ANCAs. ${ }^{9}$ Pathogenic ANCAs can provoke autoimmune responses in three ways. The first way is by impaired T cell suppression. In AAV patients, $\mathrm{CD} 4+\mathrm{CD} 25+\mathrm{T}$ cell numbers are increased, while CD4+FoxP3 $\mathrm{T}$ cells are decreased in number. Also, in GPA patients, the expression of PD-1 on circulating $\mathrm{T}$ cells is enhanced, while that on renal infiltrated $\mathrm{T}$ cells is significantly reduced. ${ }^{10,11}$ The second way is by impaired B cells suppression. CD5+ B cells producing IL-10 have regulatory function. In patients with active $\mathrm{AAV}$, the number of circulating CD5+ B cells is decreased, and normalises after remission. ${ }^{12}$ The third way is by enhanced $\mathrm{B}$ cell-stimulation by ANCA-activated neutrophil. ANCA-activated neutrophil stimulates B cells to produce ANCAs, and it releases ligands for B activating factor of TNF family (BAFF), leading to plenty of circulating ANCAs and enhanced B cell proliferation. In fact, serum BAFF levels are increased during active disease in AAV patients and decreased after remission. . $^{1314}$

Antineutrophil cytoplasmic antibodies can be generated by two assumed mechanisms: 1) Once infectious organism or medicines, which are considered perpetrators in the pathogenesis of AAV, are processed and presented by antigen-presenting cells (APCs), the release of IL-23 is enhanced and it accelerates the proliferation of Th17 cells and IL-17 production. Increased circulating IL-17 activates macrophages and drives them to secrete pro-inflammatory cytokines, resulting in priming neutrophils. MPO or PR3 in the cytoplasm of neutrophils moves to the surface or are released though lysosomes. Secreted MPO or PR3 can be recognised by APCs and presented to helper T cells. ${ }^{9,15}$ Subsequently, helper T cells can transfer the antigenic information to B cells, which produce anti-MPO or anti-PR3 ANCAs or they may differentiate to T effector memory cells, which can participate in granuloma formation. ${ }^{15}$ 2) Assuming that antigen 1 is an ANCA antigen mimicking antigen, an antibody response against antigen 1 produces antibody 1 . Also, anti-idiotypic responses against antibody 1 may produce antibody 2 . Antibody 2 can bind ANCA antigens and induce cross-reaction of priming and activating neutrophils. ${ }^{9}$

Aetiologies of AAV increase pro-inflammatory cytokines or chemokines, which, in turn, may prime neutrophils. Once neutrophils are primed, the expression of neutrophil adhesion molecules (CD11b) and Fc gamma receptors are remarkably enhanced on the surface of neutrophils; ${ }^{16}$ cytoplasmic ANCA antigens moves to the surface or they are released through lysosomes (Fig. 1A); ${ }^{15}$ and the expression of endothelial adhesion molecules, such as vascular cell adhesion molecule 1, intercellular adhesion molecule 1 and selectins, is enhanced (Fig. 1B). ${ }^{16}$ Circulating ANCA binds to the cell surface or secretory ANCA antigens and elicits "ANCA-mediated activation of neutrophils" (Fig. 1C). Thereby, the interaction between neutrophils and endothelial adhesion molecules is strengthened and induces the migration of neutrophils into adjacent tissues (Fig. 1D). Also, "ANCA-mediated activation of neutrophil" triggers the production of reactive oxygen radicals and the degranulation of neutrophils, leading to bulky inflammation on vessel walls as well as in the tissues beyond them (Fig. 1E) ${ }^{16,17}$ Alternative complement pathway can also aggravate "ANCA-mediated activation of neutrophils" by forming a loop of complement activating factors to C5a fragment (Fig. 1F).,15-17 Moreover, recently, the clinical role of neutrophil extracellular traps in association with MPO-ANCA and IL-18 in the pathogenesis of AAV has been elucidated. ${ }^{18,19}$

\section{CLASSIFICATION CRITERIA}

The American College of Rheumatology 1990 criteria for the classification of AAV (the 1990 ACR criteria) describe EGPA and GPA, but not MPA. ${ }^{3,4}$ The 1990 ACR criteria for EGPA include history of asthma, eosinophilia (peripheral eosinophil $>10 \%$ ), mono- or poly-neuropathy related to systemic vasculitis, migratory non-fixed pulmonary infiltrates, paranasal sinusitis, and extravasation of eosinophil on histology. When four of the six items are fulfilled, EGPA can be classified. ${ }^{4}$ Criteria for GPA include nasal or oral inflammation, abnormal chest radiography, urinary sediment, and granulomatous inflammation on biopsy. When two of the four items are met, GPA can be classified. ${ }^{3}$ The 1990 ACR criteria for EGPA are still reliable for use in determining EGPA. ${ }^{1,2}$ However, those for GPA are not specified, and can lead to over-classification, when biopsy-proven granuloma is not available. To overcome these limitations, the algorithm suggested by the European Medicines Agency in 2007 (the 2007 EMA algorithm) and the Chapel Hill Consensus Conference (CHCC) Nomenclature of Vasculitides proposed in 2012 (the 2012 definitions). The 2007 EMA algorithm has a flowchart in the order of EGPA, GPA, MPA, classic polyarteritis nodosa (PAN), and unclassifiable 


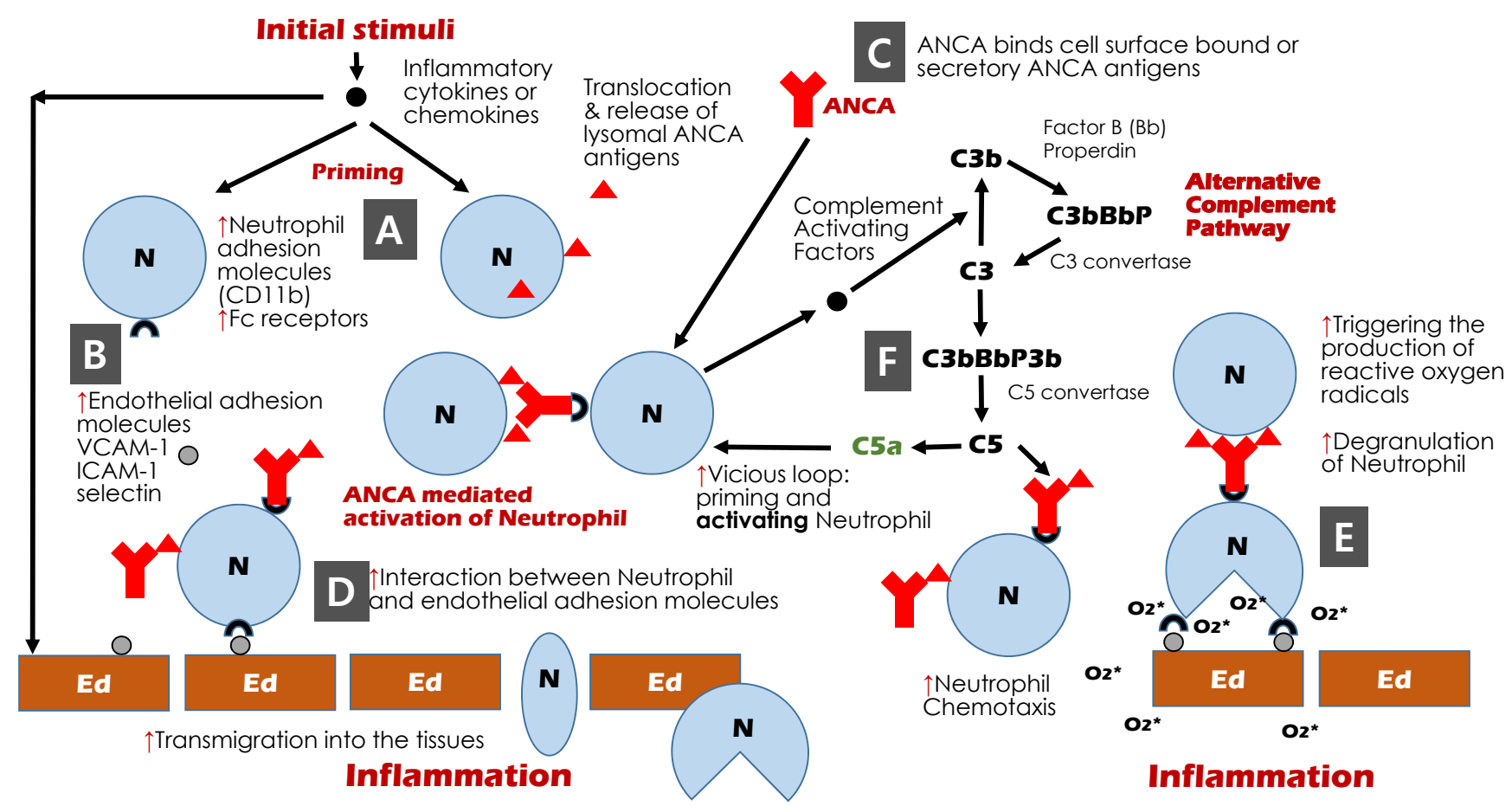

Fig. 1. Mechanism of ANCA-associated vasculitis. (A) Priming and activation of neutrophils, (B) expression of adhesion molecule on endothelial cells, (C) binding of pathogenic ANCA to ANCA-antigens, (D) interaction between neutrophils and endothelial adhesion molecules, leading to extravasation of neutrophils, (E) production of reactive oxygen radicals and degranulation of neutrophils, (F) a loop of complement activating factors to $\mathrm{C5a}$ fragment. $\mathrm{N}$, neutrophil; Ed, endothelium; CD, cluster of differentiation; VCAM-1, vascular cell adhesion molecule 1; ICAM-1, intercellular adhesion molecule 1; ANCA, antineutrophil cytoplasm antibody; $\mathrm{O}$, oxygen; $\mathrm{C}$, complement; $\mathrm{BbP}$, factor B properdin.

vasculitis, while the 2012 CHCC defines each variant of AAV with clinical and histologic features. ${ }^{1,2}$ The new concepts of PAN, based on the 2012 CHCC definitions, are that PAN never provokes glomerulonephritis and it is rarely associated with ANCA. Thus, the presence of glomerulonephritis and any type of ANCA can help to exclude PAN. ${ }^{1}$

We propose supplanted criteria, which are modifications of both the 2007 EMA algorithm and the 2012 CHCC definitions as described in Table 1. Before describing the supplemented criteria, we disclose that we used the same definitions of GPA surrogate markers and renal vasculitis, that were proposed by the 2007 EMA algorithm: GPA markers of lower respiratory tract involvement include fixed pulmonary infiltrates, nodules or cavitations and bronchial stenosis, while those for upper respiratory tract include bloody nasal discharge and crusting or ulcer, chronic sinusitis, otitis media or mastoiditis, retro-orbital mass or inflammation, subglottic stenosis, saddle nose deformity or destructive sinonasal disease. Also renal vasculitis includes haematuria [red blood cell (RBC) casts or $>10 \%$ $\mathrm{RBC}$ dysmorphism] or haematuria $\geq 2+$ or proteinuria $\geq 2+$ on urine stick. When a patient fulfils the 1990 ACR criteria for EGPA, a patient is definitely classified as EGPA. When a patient meets one of the follow three conditions, a patients is classified as GPA: 1) granulomatous and vasculitic features on histology; 2) vasculitic features without granulomatosis on histology and GPA surrogate markers; or 3) GPA surrogate markers without histology and the presence of ANCA. When a patient exhibits vasculitic features without granulomatosis on histology, but no GPA surrogate markers, or a patient has renal vasculitis and ANCA under the condition of no histology and no GPA surrogate markers, a patient is finally classified as MPA. If a patient cannot satisfy the supplemented criteria, a patient cannot be classified as AAV.

\section{RECLASSIFICATION}

PAN is occasionally difficult to be discriminated from AAV. PAN had three subgroups including cutaneous, classic, and $\mathrm{HBV}$-associated PAN.$^{20}$ However, based on the $2012 \mathrm{CHCC}$ definitions, only classic PAN can be classified as PAN. Cutaneous PAN should be reclassified as cutaneous leukocytoclastic angiitis or cutaneous arteritis, and HBV-associated PAN should be reclassified as HBV-associated vasculitis. ${ }^{1}$ Park, et al. ${ }^{21}$ applied the supplemented criteria to 32 Korean patients with PAN, who were classified according to the 1990 ACR criteria for PAN. Of 32 PAN patients, one patient was reclassified as MPA, as that patient exhibited renal vasculitis and had both MPO- and PR3-ANCA without GPA surrogate markers. Nine of the 32 PAN patients were classified as unclassifiable vasculitis, because they exhibited neither histologic and radiographic evidence of PAN nor the fulfilment of the supplemented criteria for AAV.

EGPA is rarely confused with GPA or MPA, as EGPA exhibits 


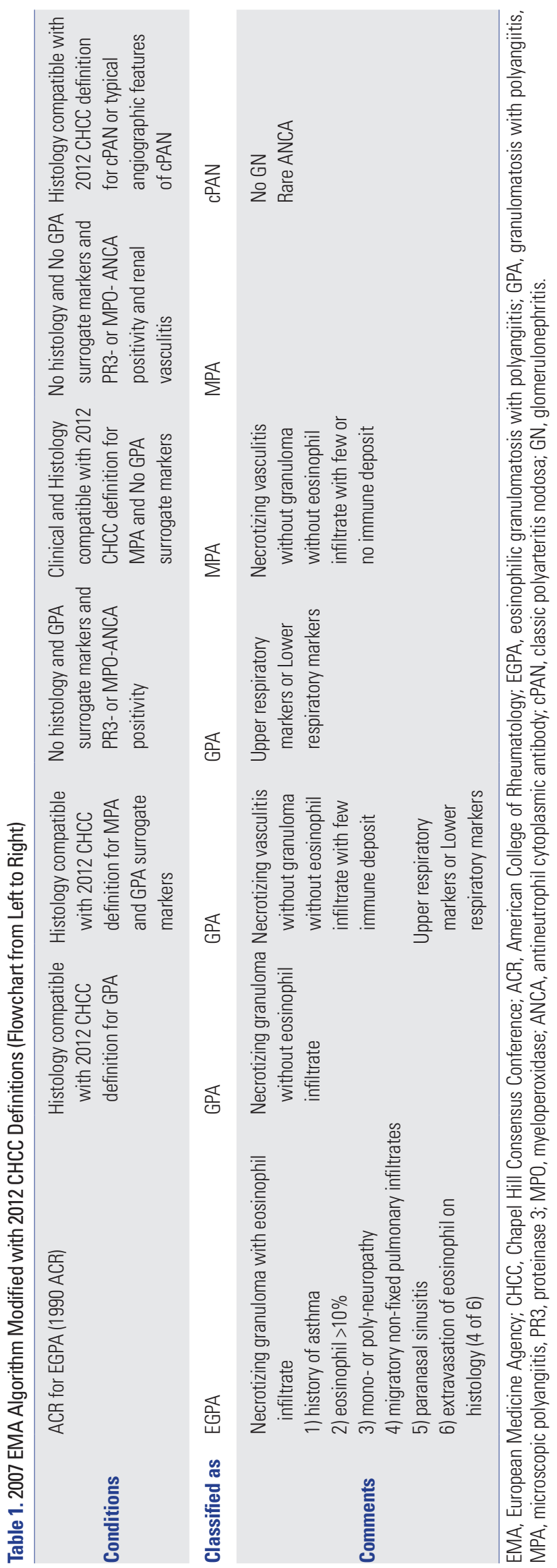

typical allergic components, along with vasculitic features. However, patients who are classified as MPA are occasionally reclassified as GPA. On the basis of our clinical experiences, a 29-year-old female patient was classified as MPA due to the presence of MPO-ANCA and renal vasculitis without GPA surrogate markers. However, subglottic inflammation and stenosis, which was incidentally detected on positron emission tomography scan, enabled her to be reclassified as GPA. A 74-year-old male patient, who was classified as MPA, could be reclassified as GPA based on cavitations and nodules in the right lung. A 72-year-old female patient with MPA could be also reclassified as GPA due to chronic and refractory otitis media. So far, final decisions on GPA have often been dependent upon histologic finding of typical granuloma. However, in the real clinical setting, the rate of detection of granuloma in patients suspected of GPA ranges from $20 \%$ to $30 \%$. Thus, we suggest that physicians may pay more attention to assessing GPA surrogate markers, when classifying patients as AAV, even in patients who were previously classified as MPA.

\section{NEW TRIALS OF RECLASSIFICATION}

Recently, a new concept for reclassification of AAV into three categories based on the presence of ANCA and its types including MPO-ANCA, PR3-ANCA and ANCA negative vasculitis was recently proposed. ${ }^{22}$ Such efforts have been motivated by several needs: First, MPA is classified by the 2007 EMA algorithm and the 2012 CHCC definitions, as MPA does not have clear classification criteria, compared to EGPA and GPA. ${ }^{1,2}$ Second, the 1990 ACR criteria for GPA and EGPA are too old to reflect currently developed AAVs. Third, neither MPO-ANCA nor PR3-ANCA was included in those criteria, despite their clinical implications. ${ }^{23}$ Moreover, recent studies have supported the distinct entities among MPO-ANCA, PR3-ANCA, and ANCA negative vasculitis. ${ }^{22,24,25}$ Even in one AAV variant, patients having MPO-ANCA can present significantly different clinical manifestations from those having PR3-ANCA or no ANCA. Furthermore, responses to immunosuppressive drugs can differ among ANCA types. Yoo, et al. ${ }^{26}$ applied this classification method to 133 Korean patients with AAV (79 MPA, 24 GPA and 30 EGPA). Ninety-one patients were reclassified as MPOANCA vasculitis, 21 as PR3-ANCA vasculitis and 21 as ANCA negative vasculitis. MPA patients were mainly reclassified as MPO-ANCA vasculitis (82.4\%), GPA patients as PR3-ANCA vasculitis (71.4\%), and EGPA patients as ANCA negative vasculitis (76.2\%). However, ANCA positivity or the type of ANCA had no influence on outcomes of AAV.

The Diagnostic and Classification Criteria for Primary Systemic Vasculitis (DCVAS) and collaborators recently proposed the ACR/EULAR (The European League Against Rheumatism) 2017 provisional classification criteria for GPA. These criteria include nine items, of which five items are clinical vari- 
Table 2. The ACR/EULAR 2017 Provisional Classification Criteria for GPA

\begin{tabular}{lc}
\multicolumn{1}{c}{ Items } & Score \\
\hline $\begin{array}{l}\text { Score for the ACR/EULAR } 2017 \text { provisional classification } \\
\text { criteria for GPA }\end{array}$ & Sum $\geq 5$ \\
Bloody nasal discharge, ulcers, crusting or sinonasal congestion & 3 \\
Nasal polyps & -4 \\
Hearing loss or reduction & 1 \\
Cartilaginous involvement & 2 \\
Red or painful eyes & 1 \\
C-ANCA or PR3-ANCA & 5 \\
Eosinophil count $\geq 1\left(\times 10^{9} / L\right)$ & -3 \\
Nodule, mass or cavitation on chest imaging & 2 \\
Granuloma on biopsy & 3 \\
\hline
\end{tabular}

ACR, American College of Rheumatology; EULAR, European League Against Rheumatism; GPA, granulomatosis with polyangiitis; C-ANCA, cytoplasmic antineutrophil cytoplasmic antibody; PR3-ANCA, proteinase 3-ANCA.

ables and four items are test variables. Different weights are assigned to each item as shown in Table 2. The score assigned to C-ANCA or PR3-ANCA is the highest among the items. Particularly, nasal polyps and eosinophilia $>10 \%$ are major negative contributors to the diagnosis of GPA. When the sum of scores is 5 or greater, patients can be classified as GPA (presented at 2016 ACR session: New Classification Criteria for ANCA-associated Vasculitis: implications for clinical practice). Yoo, et al. ${ }^{27}$ applied the 2017 provisional criteria suggested by DCVAS to 150 Korean patients with AAV. Three patients, who had been previously classified as GPA, were reclassified as no GPA. All 3 patients had definite histologic evidence of granuloma, but they fulfilled no more items. They were not reclassified as MPA due to granuloma or EGPA due to no history of asthma, eosinophilia or eosinophilic extravasation. Moreover, the 2017 provisional criteria has several limitations that they include no item of kidney involvement for GPA and an item of fixed lung infiltrates is excluded. Meanwhile, one EGPA patient was reclassified as GPA due to sinonasal congestion (3) and PR3-ANCA positivity (5), despite eosinophilia $(-3)$. In this case, PR3-ANCA positivity might be overestimated compared to the 1990 ACR criteria or the 2007 EMA algorithm modified by the 2012 CHCC definitions. Thus, the weight of PR3-ANCA positivity should be readjusted in the near future. In addition, 5 MPA patients were reclassified as GPA and PR3ANCA also played a major role in the classification of GPA in 2 patients. ${ }^{27}$

\section{ASSESSMENT OF DISEASE ACTIVITY AND DEFINTIONS OF PROGNOSIS}

The disease activity of AAV can be mainly assessed by Birmingham vasculitis activity score (BVAS) (or BVAS for GPA) ${ }^{28,29}$ and five factor scores [FFS (1996) and FFS (2009)]. ${ }^{30}$ Damage derived from AAV can be assessed using vasculitis damage in- dex (VDI) and combined damage assessment. ${ }^{31}$ Functions of patients AAV are evaluated by generalised methods, such as SF-36. ${ }^{32}$ BVAS is the most widely used to assess vasculitis activity in AAV. BVAS is composed of nine major items (plus one item of others) including general, cutaneous, mucous membrane/eyes, ear nose throat (ENT), chest (pulmonary), cardiovascular, abdominal, renal and nervous system involvements. The score assigned to the same sub-items can be different depending on the duration and severity of symptoms, such as persistent and newly developed or worsening. ${ }^{28}$ The FFS proposed in 1996 includes elevated serum creatinine, proteinuria, severe gastrointestinal (GI) tract involvement, cardiomyopathy and CNS involvement. Meanwhile, the modified version of FFS proposed in 2009 consists of age $>65$ years, insufficient renal function, severe GI tract involvement, cardiomyopathy and no ENT symptoms. ${ }^{30}$ Remission is defined as absence of disease activity attributable to active disease requiring the maintenance of immunosuppressive drugs. Relapse is defined as recurrence or new onset of active disease. Refractory disease is defined as unchanged or increased disease activity in acute vasculitis after 4 weeks of treatment or lack of response or chronic and persistent disease after over 12 weeks of treatment. ${ }^{33}$

\section{PREDICTORS AT DIAGNOSIS OF PROGNOSIS}

In terms of MPA, Oh, et al. ${ }^{34}$ first reported the association variables or indices at diagnosis and prognosis in Korean patients with MPA in 2009. Eighteen MPA patients were reviewed, and 10 patients had died. The most common cause of death was diffuse alveolar haemorrhage. BVAS $\geq 19$, FFS $\geq 1$, age $\geq 66$ years and diffuse alveolar haemorrhage at diagnosis predicted all-cause mortality in MPA patients. Ahn, et al. ${ }^{35}$ also reported the results of the similar study in 55 Korean patients with MPA. In this study, instead of FFS and diffuse alveolar haemorrhage, cardiomyopathy and interstitial lung disease along with BVAS $>9$ and old age were predictors of all-cause mortality. In 2017, Oh, et al. ${ }^{36}$ reviewed the medical records of 90 Korean patients with MPA, and investigated the association between initial indices and relapse during the follow-up. They insisted that chest (pulmonary) and renal involvement, BVAS $\geq 13.5$, and FFS (1996) $\geq 1$ at the time of diagnosis were independent predictors of relapse of MPA. So far, MPO-ANCA and PR3-ANCA have been considered to be associated with mortality and relapse in MPA patients, respectively. ${ }^{37,38}$ However, ANCA positivity has not been proven as a predictor of poor prognosis in Korean patients with MPA.

In terms of GPA, Kim, et al. ${ }^{39}$ reported concrete data regarding clinicopathologic characteristics of Korean patients with GPA. Besides Koran patients with MPA, initial ANCA positivity was significantly associated with renal involvement of GPA and all-cause mortality during the follow-up. Moreover, pa- 
tients with ANCA exhibited higher initial BVAS for GPA than those without, and PR3-ANCA mainly contributed to the initial BVAS for GPA. They also compared GPA patients according to histologic patterns and granulomatous and vasculitic types. Patients with the granulomatous type exhibited young age and low frequency of renal involvement, compared to those with the vasculitic type. Yoo, et al. ${ }^{40}$ evaluated the predictors of refractory disease during the follow-up in Korean patients with GPA. The optimal cut-offs of BVAS for GPA, and FFS (1996) and FFS (2009) at diagnosis to predict refractory disease of GPA were 9.5, 2, and 2 respectively. In Kaplan-Meier survival analysis, initial BVAS $\geq 9.5$, FFS (1996) $\geq 2$, and FFS (2009) $\geq 2$ meaningfully predicted cumulative refractory disease free survival. However, in multivariable Cox hazard model, only BVAS at diagnosis $\geq 9.5$ was identified as an independent predictor of refractory disease in Korean patients with GPA.

In terms of EGPA, Kim, et al. ${ }^{41}$ analysed the clinical features and prognosis in Korean patients with EGPA. They divided EGPA patients according to either ANCA positivity or responses to treatment and compared organ involvements between the two groups. Between patients with and without ANCA at diagnosis, ANCA-negative patients less frequently exhibited renal involvement. Also, older age, diagnosis at earlier stage, less cutaneous involvement, more respiratory involvement, high C-reactive protein (CRP), and more generalized symptoms were observed in good responders. Meanwhile, in another study, Kim, et al. ${ }^{42}$ investigated initial variables associated with relapse during follow-up in Korean patients with EGPA. They first obtained the optimal cut-off of FFS to predict relapse of EGPA as 1. When they divided EGPA patients according to FFS of 1 , relapse was identified more frequently in patients with $\mathrm{FFS} \geq 1$ than those with $\mathrm{FFS}<1$ (68.8\% vs. $7.1 \%)$.

\section{MORTALITY}

In Western countries, 1-year-, 5-year-, and 10-year-cumulative patient survival rates have been reported as $82-95 \%, 74-97 \%$, and $89 \%$, respectively. ${ }^{43}$ In East Asian countries with similar ethnicities to Korea, 1-year- and 5-year-cumulative patient survival rates were reported at $79.1 \%$ and $63.6 \%$ in Japan, and 6-month-cumulative patient survival rate was estimated as $78.7 \%$ in China. ${ }^{44,45}$ In Korea, there have been three reports on survival rate in AAV patients before 2016. The first study reported on all-cause mortality in MPA patients with a rate of $56 \%{ }^{34}$ The second study reported that 1-year- and 3-year-cumulative survival rates were $93.9 \%$ and $89.2 \%$ in MPA patients. ${ }^{35}$ The third study reported that $22.2 \%$ of GPA patients died during follow-up. ${ }^{39}$ In 2016, Lee, et al. ${ }^{46}$ investigated long term prognosis in 48 Korean patients with renal vasculitis. Six patients had no ANCAs. In Kaplan-Meier survival analysis, ANCA negativity was a significant predictor of renal survival, but not patient survival. However, in the multivariable Cox hazards model, ANCA negativity did not affect cumulative renal and patient survivals. In 2018, Mun, et al. ${ }^{47}$ investigated the initial predictors of all-cause mortality in 153 Korean patients with AAV. Fourteen patients (9.2\%) (7 MPA and 7 GPA patients) died during the mean follow-up of 56.9 months. Seven patients died of bacterial, viral, or fungal pneumonia, and 3 patients died of sepsis. In the remaining patients, cardiac arrest of unknown aetiology, alveolar haemorrhage, H1N1 influenza and biliary infection were causes of mortality. One-year-, 5-year-, and 10-year-cumulative patient survival rates were calculated as 96.1, 94.8, and 92.8\% in patients with AAV. In the KaplanMeier survival analysis, FFS (2009) $\geq 2$, chronic kidney disease stage $\geq 3$ or end stage renal disease (ESRD), and diffuse alveolar haemorrhage were associated with all-cause mortality in AAV patients. However, in the multivariable Cox hazards model, only FFS (2009) $\geq 2$ (HR 16.5) and diffuse alveolar haemorrhage (HR 3.7) at the time of diagnosis were left as independent predictors of all-cause mortality.

\section{SERIOUS INFECTION}

Serious infections that require hospitalisation have been reported to occur in $20 \%$ to $60 \%$ of patients with AAV. The wide variability in serious infection rate may stem from AAV variants, ethnic and geographical differences, antibiotics-prophylaxis and the follow-up duration. ${ }^{48,49}$ In addition, old age, ${ }^{50} \mathrm{im}$ munosuppressive drugs, ${ }^{51-53}$ reduced renal function, ${ }^{54}$ and no ANCA have been found to be related to serious infections. ${ }^{55}$ However, most clinical studies regarding serious infection have been conducted in Western countries and only a few observational studies have been undertaken in Korea. Kim, et al. ${ }^{39}$ reported 20 of 45 Korean patients with GPA (44.4\%) suffered from various infections, including pneumonia, bacteraemia, septic shock, peritonitis, and so on. Yoo, et al. ${ }^{56}$ reported retrospective data on hospitalised infections in 154 Korean patients with AAV. Forty-four of the 154 patients (28.6\%) had been admitted for serious infection. One-year-, 5-year-, and 10-yearcumulative hospitalised infection free survival rates were 85.1, 77.9 and $72.7 \%$, respectively. Of 44 patients, there were 56 episodes of hospitalised infection and the most common aetiology was pneumonia (29 episodes), followed by aspergillosis ( 6 episodes), biliary infection (4 episodes), and pneumocystis jirovecii infection (4 episodes). In the Kaplan-Meier analysis, BVAS at diagnosis $\geq 20.5$, FFS (2009) at diagnosis $\geq 1.5$, pulmonary manifestation, renal manifestation, MPO-ANCA (or PANCA) and ANCA positivity were remarkably associated with hospitalised infection. However, in the multiple Cox hazards model analysis, only BVAS at diagnosis $\geq 20.5$ and pulmonary involvement were predictors of serious infection. 


\section{CANCERS}

So far, various autoimmune diseases with chronic inflammatory burdens have been shown to be associated with an increase in the development of cancers. Among autoimmune diseases, rheumatoid arthritis (RA), systemic lupus erythematosus, Sjogren syndrome, systemic sclerosis, and idiopathic inflammatory myopathies are well-known to be linked to cancer occurrence. ${ }^{57-60}$ No only autoimmune systemic inflammation driven by AAV, but also immunosuppressive drugs for AAV may impair immune surveillance and recognitions of cancer cells or act as direct oncogenic aetiology. ${ }^{61,62}$ Cancers occur more frequently in AAV patients than the general population [standardised incidence ratios (SIRs) from 1.6 to 2.4] ${ }^{63-66}$ Common cancer types are urinary tract cancer, skin cancer and haematological cancers. ${ }^{63-65}$ The overall SIR of cancer in AAV patients, however, tends to be reduced due to more safe therapeutic regimens and decreased exposure to both AAV itself and oncogenic immunosuppressive drugs. ${ }^{67}$ Yoo, et al ${ }^{68}$ first provided the incidence and types of cancers in 150 Korean patients with AAV (81 MPA, 38 GPA, and 31 EGPA). Four of 150 AAV patients (2.7\%) had cancers with the mean follow-up duration of 50.7 months. Each AAV patients exhibited four different cancers, including gastric cancer, lung cancer, prostate cancer, and Non-Hodgkin lymphoma. The gap-time from diagnosis of AAV to cancer development was less than 1 year in two patients. The overall SIR of cancer in AAV patients was 1.43 compared to the general Korean population, but it was not statistically significant. Age at diagnosis of cancer, gender, and AAV variants did not influence SIRs.

\section{INDICES AT DIAGNOSIS FOR ACTIVITY AND PROGNOSIS}

We have discovered that initial vasculitis activity, prognostic factors, and organ-involvement at diagnosis could be associated with the prognosis of AAV. Here, three laboratory variables to estimate the current vasculitis activity, as well as outcomes of AAV, will be introduced. The advantage of the use thereof is that they are currently measured in routine blood tests. First, the consumption of neutrophils can increase the number of immature granulocytes, which is called a granulocytic shift to the left. ${ }^{69}$ Delta neutrophil index (DNI) is a leukocyte sub-fraction, which is automatically measured by subtracting the fraction of mature polymorphonuclear leukocytes from the sum of MPO-reactive cells. ${ }^{70} \mathrm{DNI}$ has been reported to be associated with conditions, in which mature neutrophils are consumed, such as sepsis and disseminated intravascular coagulation. ${ }^{71}$ Recently, Yoo, et al. ${ }^{72}$ suggested a association between DNI and either current BVAS or relapse in 97 Korean patients with AAV. They indicated that DNI could reflect current BVAS and that DNI $\geq 0.65 \%$ could predict a higher rate of relapse during follow-up of AAV. Second, RBC distribution width (RDW) is a value of heterogeneity in the size of circulating RBCs. RDW is clinically used to discriminate various types of anaemia. ${ }^{73}$ In the meantime, RDW has been considered to be linked to inflammatory burden in systemic rheumatic diseases. ${ }^{7475} \mathrm{Kim}$, et al. ${ }^{76}$ reported that RDW was correlated with current vasculitis activity only in GPA patients among $150 \mathrm{Ko}-$ rean patients with AAV. In the Kaplan-Meier survival analysis, RDW $\geq 15.4 \%$ and absence of ENT manifestations were associated with a higher incidence of refractory disease in GPA. Furthermore, in the multivariable Cox hazards analysis, only RDW was an independent predictor of refractory disease of GPA during follow-up. Third, mean platelet volume (MPV) is a marker to reflect platelet distributions in the peripheral circulation. MPV increases as platelet production increases and decreases with reduced production of platelets. ${ }^{77}$ MPV exhibits a biphasic pattern in that MPA mainly increases in low-grade inflammation, while it often decreases in high-grade inflammation. ${ }^{78} \mathrm{Kim}$, et al. ${ }^{79}$ elucidated that MPV was independently and inversely associated with current BVAS in multivariate linear regression analysis in 88 Korean patients with MPA. They also provided the optimal cut-off of MPV for predicting severe MPA (MPV $\leq 8.85 \mathrm{fL}$ ) and demonstrated that MPV increased along with improvement of vasculitis activity.

In addition, several ratios consisting of two or three laboratory variables at diagnosis of AAV have been proposed for assessing vasculitis activity at diagnosis as well as prognosis during follow-up. Kim, et al. ${ }^{80}$ applied systemic immune-inflammation index (SII) [SII=platelet count $\times$ neutrophil count/lymphocyte count] to AAV patients. They elucidated that SII at diagnosis $\geq 1573.56$ could estimate severe AAV at diagnosis and predict the poor outcome in AAV patients. Ahn, et al. ${ }^{81}$ proved that neutrophil to lymphocyte ratio and albumin to globulin ratio might be useful for assessing the current activity and relapse in AAV patients. Park, et al. ${ }^{82}$ also provided evidence that platelet to lymphocyte ratio might be associated with the current activity of AAV. Furthermore, it has been recently reported that albumin to globulin ratio was inversely associated with all-cause mortality in MPA patients, and CRP to serum albumin ratio could be a risk factor for all-cause mortality in AAV patients. ${ }^{83,84}$ On the other hands, in the pathogenesis of AAV, primed and activated neutrophils release complement-activating factors and they, in turn, cleave complement 3 (C3) into C3a and $3 \mathrm{Cb} .{ }^{85}$ Thus, it could be assumed that serum C3 level may be negatively correlated with AAV activity. Choi, et al. ${ }^{86}$ clarified that serum C3 level $<90 \mathrm{mg} / \mathrm{dL}$ was associated with both activity and poor prognosis of AAV.

\section{OTHERS}

\section{Cardiac function}

Cardiovascular manifestations as an item of BVAS consists of 
loss of pulses, valvular heart disease, pericarditis, ischaemic cardiac pain (or ischaemic heart disease), cardiomyopathy and congestive heart failure. ${ }^{28,29}$ Cardiovascular manifestations occasionally occur in AAV, and its incidence rates differ for both AAV variants and ANCA types (5-49\%). ${ }^{23}$ Initial involvement of the heart was reported to be related to poor prognosis..$^{30,87}$ Recently, Ahn, et al. ${ }^{88}$ reported the results of echocardiography performed 3 months before and after diagnosis of AAV in 89 Korean patients. They compared echocardiographic results in AAV patients with those in age- and gender-matched healthy controls. AAV patients showed lower mean left ventricular ejection fraction (LVEF) than controls (64.0\% vs. $69.1 \%$ ). They also exhibited left ventricular diastolic dysfunction more frequently than controls with a higher mean E/E' ratio (11.7 vs. 8.8). Furthermore, measuring right ventricular systolic pressure (RVSP), which can estimate the presence of pulmonary arterial hypertension, the authors indicated that MPA and EGPA patients, but not GPA patients, showed enhanced RVSP than controls. However, there were no significant differences in LVEF, E/E' ratio and RVSP among patients with MPA, GPA and EGPA. ${ }^{88}$ Physicians should be encouraged to perform echocardiography within 3 months after diagnosis of AAV.

\section{Outcome of kidney transplantation}

Renal involvement frequently occurs in AAV patients (90$100 \%$ in MPA, $50-80 \%$ in GPA, $31-51 \%$ in ANCA positive EGPA, and $4-16 \%$ in ANCA negative EGPA) ${ }^{23}$ Pauci-immune crescentic and necrotising glomerulonephritis are the typical histological findings. ${ }^{89}$ Renal involvement of AAV may rapidly progress to ESRD in up to $40 \%$ of patients. ${ }^{90,91}$ In these cases, kidney transplantation may be more valuable than dialysis maintenance. ${ }^{92,93}$ So far, histological features, age, PR3-ANCA positivity, and creatinine clearance rate at kidney transplantation were reported to be poor prognostic factors. ${ }^{94}$ Recently, Park, et al ${ }^{95}$ first reported the outcome of kidney transplantation in 144 Korean patients with AAV. Of 144 AAV patients, four AAV patients ( 3 female patients with MPA and 1 male patient with EGPA) underwent kidney transplantation, and they were followed up for more than 2 years. Time-gap from diagnosis of AAV to ESRD ranged from 1 to 48 months and that from AAV to kidney transplantation was 2 to 95 months. One kidney recipient with MPA underwent renal biopsy three times due to renal dysfunction. Renal histology revealed rejection rather than relapse of MPA. There was no relapse or graft failure in the four Korean kidney recipients with AAV. The authors concluded that renal outcomes of kidney transplantation was good and that kidney transplantation could be recommended as a safe and efficient therapeutic modality in patients with ESRD related to $\mathrm{AAV}^{95}$

\section{Rheumatoid factor false positive}

Rheumatoid factor (RF) is a typical antibody in RA and it can increase the accuracy of the classification as RA. ${ }^{96}$ However, $\mathrm{RF}$ can be falsely detected in various medical conditions amplifying immunoglobulin $\mathrm{G}(\mathrm{IgG})$ production. ${ }^{97} \mathrm{AAV}$ is also a medical condition augmenting the production of IgG, particularly ANCAs. Thus, it can be reasonably speculated that RF can be frequently detected in AAV patients. Moon, et al ${ }^{98}$ reviewed the medical records and laboratory data 128 Korean patients without RA at the time of diagnosis of AAV and counted the number of patients with RF. The rate of RF false positivity was $39.1 \%$ in AAV patients. In multivariable analysis, cutaneous manifestation was the only independent predictor of RF false positivity in EGPA patients, but not in MPA and GPA patients. They explained the link between cutaneous manifestation and RF false positivity via three hypotheses: First, combination of RF with cryoglobulin is associated with skin vasculitis. ${ }^{99}$ Second, immune-complex depiction of IgG-RF and complement 3 in the dermal-epidermal junction drives dermatitis. ${ }^{100}$ Third, IgA-RF immune complex induces silent dermatitis. ${ }^{101}$ Also, they explained the link between RF-induced dermatitis and EGPA by the potential association between RF and eosinophilia, leading to eosinophilic pneumonia. ${ }^{102} \mathrm{How}-$ ever, RF false positivity did not have an influence on the outcome of AAV or the development of RA. ${ }^{98}$

\section{Liver related issues}

Although liver is one of the major organs with plenty of capillaries like lungs and kidneys, hepatic involvement of AAV might have been underestimated due to non-specific symptoms. ${ }^{103}$ However, AAV can theoretically induce the hepatocellular damages and enhance the potential of liver fibrosis similar to autoimmune hepatitis and primary biliary cirrhosis. ${ }^{104,105}$ For predicting liver fibrosis, aspartate aminotransferase to platelet ratio index (APRI) and an index of fibrosis (FIB-4) are widely used. ${ }^{106}$ Lee, et al ${ }^{107}$ recently reported that AAV may provoke subclinical but significant liver fibrosis at diagnosis using APRI and FIB- 4 , and suggested FFS $\geq 1$ at diagnosis as a predictor of liver fibrosis. Furthermore, they also reported that resolved HBV infection could influence vasculitis at diagnosis and relapse after remission in EGPA patients. ${ }^{108}$

\section{CONCLUSIONS AND FUTURE STUDIES}

So far, there have been noticeable advancement in studies on pathophysiology of AAV and the classification criteria for AAV in Western countries. However, studies analysing clinical features of Korean patients with AAV have only been conducted and reported since 2000. Nevertheless, Korean physicians have rapidly adapted to world-wide changes, and have applied new concepts and classification criteria to Korean patients with AAV. In this review, we found several major features in Korean patients with AAV. First, initial vasculitis activity, prognostic factor score, age and specific organ-involvement 
seem to be associated with either all-cause mortality or poor disease course. ANCA positivity appears to be related to mortality only in GPA. Second, the overall mortality rate during follow-up of AAV has been reported to be 9.2\%. Furthermore, 1-year-, 5-year-, and 10-year-cumulative survival rates were reported as $96.1,94.8$, and $92.8 \%$ in a considerable number of AAV patients. Third, the rate of serious infection was described as $28.6 \%$ and 1-year, 5-year-, and 10-year-cumulative hospitalised infection free survival rates ranged $85.1 \%$ to $72.7 \%$. Fourth, the overall SIR of cancer in AAV patients appeared to be 1.43, compared to the general Korean population.

Nevertheless, previous studies analysing clinical features and prognosis in Korean patients with AAV have several limitations of a retrospective study-design and a small number of patients. Furthermore, there is no nation-wide cohort of AAV in Korea yet. Thus, a prospective future studies with a larger number of patients by establishing a Korean nation-wide cohort of AAV patients will provide more valuable epidemiologic information and the opportunity to participate in international clinical trials.

\section{ORCID iDs}

Chan-Bum Choi https://orcid.org/0000-0002-4691-5455

Yong-Beom Park https://orcid.org/0000-0003-4695-8620

Sang-Won Lee https://orcid.org/0000-0002-8038-3341

\section{REFERENCES}

1. Jennette JC, Falk RJ, Bacon PA, Basu N, Cid MC, Ferrario F, et al. 2012 revised International Chapel Hill Consensus Conference Nomenclature of Vasculitides. Arthritis Rheum 2013;65:1-11.

2. Watts R, Lane S, Hanslik T, Hauser T, Hellmich B, Koldingsnes W, et al. Development and validation of a consensus methodology for the classification of the ANCA-associated vasculitides and polyarteritis nodosa for epidemiological studies. Ann Rheum Dis 2007;66:222-7.

3. Leavitt RY, Fauci AS, Bloch DA, Michel BA, Hunder GG, Arend WP, et al. The American College of Rheumatology 1990 criteria for the classification of Wegener's granulomatosis. Arthritis Rheum 1990;33:1101-7.

4. Masi AT, Hunder GG, Lie JT, Michel BA, Bloch DA, Arend WP, et al. The American College of Rheumatology 1990 criteria for the classification of Churg-Strauss syndrome (allergic granulomatosis and angiitis). Arthritis Rheum 1990;33:1094-100.

5. Park YB, Kim JY, Linton JA, Jung HJ, Lee SK, Shin DH. Clinicopathologic study of Wegener's granulomatosis with special emphasis on early lesions in 10 Korean patients. Yonsei Med J 2001;42:46-54.

6. Kim HW, Song YW. ANCA-associated vasculitis: report from Korea. Clin Exp Nephrol 2013;17:708-11.

7. Jennette JC, Falk RJ, Hu P, Xiao H. Pathogenesis of antineutrophil cytoplasmic autoantibody-associated small-vessel vasculitis. Annu Rev Pathol 2013;8:139-60.

8. Xu PC, Cui Z, Chen M, Hellmark T, Zhao MH. Comparison of characteristics of natural autoantibodies against myeloperoxidase and anti-myeloperoxidase autoantibodies from patients with microscopic polyangiitis. Rheumatology (Oxford) 2011;50:
1236-43.

9. Jennette JC, Falk RJ. Pathogenesis of antineutrophil cytoplasmic autoantibody-mediated disease. Nat Rev Rheumatol 2014;10:463-73.

10. Free ME, Bunch DO, McGregor JA, Jones BE, Berg EA, Hogan SL, et al. Patients with antineutrophil cytoplasmic antibody-associated vasculitis have defective Treg cell function exacerbated by the presence of a suppression-resistant effector cell population. Arthritis Rheum 2013;65:1922-33.

11. von Borstel A, Sanders JS, Rutgers A, Stegeman CA, Heeringa P, Abdulahad WH. Cellular immune regulation in the pathogenesis of ANCA-associated vasculitides. Autoimmun Rev 2018;17:413-21.

12. Unizony S, Lim N, Phippard DJ, Carey VJ, Miloslavsky EM, Tchao $\mathrm{NK}$, et al. Peripheral CD5+ B cells in antineutrophil cytoplasmic antibody-associated vasculitis. Arthritis Rheumatol 2015;67:535-44.

13. Jennette JC, Falk RJ. B cell-mediated pathogenesis of ANCA-mediated vasculitis. Semin Immunopathol 2014;36:327-38.

14. Nagai M, Hirayama K, Ebihara I, Shimohata H, Kobayashi M, Koyama A. Serum levels of BAFF and APRIL in myeloperoxidase anti-neutrophil cytoplasmic autoantibody-associated renal vasculitis: association with disease activity. Nephron Clin Pract 2011; 118:c339-45.

15. Chen M, Kallenberg CG. ANCA-associated vasculitides--advances in pathogenesis and treatment. Nat Rev Rheumatol 2010;6:653-64.

16. Kallenberg CG, Heeringa P, Stegeman CA. Mechanisms of Disease: pathogenesis and treatment of ANCA-associated vasculitides. Nat Clin Pract Rheumatol 2006;2:661-70.

17. Kallenberg CG, Stegeman CA, Abdulahad WH, Heeringa P. Pathogenesis of ANCA-associated vasculitis: new possibilities for intervention. Am J Kidney Dis 2013;62:1176-87.

18. Nakazawa D, Shida H, Tomaru U, Yoshida M, Nishio S, Atsumi T, et al. Enhanced formation and disordered regulation of NETs in myeloperoxidase-ANCA-associated microscopic polyangiitis. J Am Soc Nephrol 2014;25:990-7.

19. Lee KH, Kronbichler A, Park DD, Park Y, Moon H, Kim H, et al. Neutrophil extracellular traps (NETs) in autoimmune diseases: a comprehensive review. Autoimmun Rev 2017;16:1160-73.

20. Lightfoot RW Jr, Michel BA, Bloch DA, Hunder GG, Zvaifler NJ, McShane DJ, et al. The American College of Rheumatology 1990 criteria for the classification of polyarteritis nodosa. Arthritis Rheum 1990;33:1088-93.

21. Park ES, Ahn SS, Jung SM, Song JJ, Park YB, Lee SW. Reclassification of polyarteritis nodosa based on the 1990 ACR criteria using the 2007 EMA algorithm modified by the 2012 CHCC definitions. Clin Exp Rheumatol 2018;36 Suppl 111:165-6.

22. Cornec D, Cornec-Le Gall E, Fervenza FC, Specks U. ANCA-associated vasculitis - clinical utility of using ANCA specificity to classify patients. Nat Rev Rheumatol 2016;12:570-9.

23. Millet A, Pederzoli-Ribeil M, Guillevin L, Witko-Sarsat V, Mouthon L. Antineutrophil cytoplasmic antibody-associated vasculitides: is it time to split up the group? Ann Rheum Dis 2013; 72:1273-9.

24. Sokolowska BM, Szczeklik WK, Wludarczyk AA, Kuczia PP, Jakiela BA, Gasior JA, et al. ANCA-positive and ANCA-negative phenotypes of eosinophilic granulomatosis with polyangiitis (EGPA): outcome and long-term follow-up of 50 patients from a single Polish center. Clin Exp Rheumatol 2014;32(3 Suppl 82): S41-7.

25. Kallenberg CG. Pathogenesis of ANCA-associated vasculitis, an update. Clin Rev Allergy Immunol 2011;41:224-31.

26. Yoo J, Kim HJ, Ahn SS, Jung SM, Song JJ, Park YB, et al. Clinical and prognostic features of Korean patients with MPO-ANCA, PR3-ANCA and ANCA-negative vasculitis. Clin Exp Rheumatol 2017;35 Suppl 103:111-8. 
27. Yoo J, Kim HJ, Ahn SS, Jung SM, Song JJ, Park YB, et al. The utility of the ACR/EULAR 2017 provisional classification criteria for granulomatosis with polyangiitis in Korean patients with antineutrophil cytoplasmic antibody-associated vasculitis. Clin Exp Rheumatol 2018;36 Suppl 111:85-7.

28. Mukhtyar C, Lee R, Brown D, Carruthers D, Dasgupta B, Dubey $\mathrm{S}$, et al. Modification and validation of the Birmingham Vasculitis Activity Score (version 3). Ann Rheum Dis 2009;68:1827-32.

29. Stone JH, Hoffman GS, Merkel PA, Min YI, Uhlfelder ML, Hellmann DB, et al. A disease-specific activity index for Wegener's granulomatosis: modification of the Birmingham Vasculitis Activity Score. International Network for the Study of the Systemic Vasculitides (INSSYS). Arthritis Rheum 2001;44:912-20.

30. Guillevin L, Pagnoux C, Seror R, Mahr A, Mouthon L, Le Toumelin P; French Vasculitis Study Group (FVSG). The Five-Factor Score revisited: assessment of prognoses of systemic necrotizing vasculitides based on the French Vasculitis Study Group (FVSG) cohort. Medicine (Baltimore) 2011;90:19-27.

31. Exley AR, Bacon PA, Luqmani RA, Kitas GD, Gordon C, Savage $\mathrm{CO}$, et al. Development and initial validation of the Vasculitis Damage Index for the standardized clinical assessment of damage in the systemic vasculitides. Arthritis Rheum 1997;40:371-80.

32. Ware JE Jr, Sherbourne CD. The MOS 36-item short-form health survey (SF-36). I. Conceptual framework and item selection. Med Care 1992;30:473-83.

33. Mukhtyar C, Hellmich B, Jayne D, Flossmann O, Luqmani R. Remission in antineutrophil cytoplasmic antibody-associated systemic vasculitis. Clin Exp Rheumatol 2006;24(6 Suppl 43):S-93-8.

34. Oh JS, Lee CK, Kim YG, Nah SS, Moon HB, Yoo B. Clinical features and outcomes of microscopic polyangiitis in Korea. J Korean Med Sci 2009;24:269-74.

35. Ahn JK, Hwang JW, Lee J, Jeon CH, Cha HS, Koh EM. Clinical features and outcome of microscopic polyangiitis under a new consensus algorithm of ANCA-associated vasculitides in Korea. Rheumatol Int 2012;32:2979-86.

36. Oh YJ, Ahn SS, Park ES, Jung SM, Song JJ, Park YB, et al. Chest and renal involvements, Birmingham vascular activity score more than 13.5 and five factor score (1996) more than 1 at diagnosis are significant predictors of relapse of microscopic polyangiitis. Clin Exp Rheumatol 2017;35 Suppl 103:47-54.

37. Flossmann O, Berden A, de Groot K, Hagen C, Harper L, Heijl C, et al. Long-term patient survival in ANCA-associated vasculitis. Ann Rheum Dis 2011;70:488-94.

38. Lionaki S, Blyth ER, Hogan SL, Hu Y, Senior BA, Jennette CE, et al. Classification of antineutrophil cytoplasmic autoantibody vasculitides: the role of antineutrophil cytoplasmic autoantibody specificity for myeloperoxidase or proteinase 3 in disease recognition and prognosis. Arthritis Rheum 2012;64:3452-62.

39. Kim HW, Kim JW, Im CH, Shin KC, Lee EY, Lee EB, et al. The clinicopathologic characteristics of granulomatosis with polyangiitis (Wegener's): a retrospective study of 45 patients in Korea. Mod Rheumatol 2013;23:864-71.

40. Yoo J, Kim HJ, Jung SM, Song JJ, Park YB, Lee SW. Birmingham vasculitis activity score of more than 9.5 at diagnosis is an independent predictor of refractory disease in granulomatosis with polyangiitis. Int J Rheum Dis 2017;20:1593-605.

41. Kim MY, Sohn KH, Song WJ, Park HW, Cho SH, Min KU, et al. Clinical features and prognostic factors of Churg-Strauss syndrome. Korean J Intern Med 2014;29:85-95.

42. Kim DS, Song JJ, Park YB, Lee SW. Five factor score of more than 1 is associated with relapse during the first 2 year-follow up in patients with eosinophilic granulomatosis with polyangiitis. Int J Rheum Dis 2017;20:1261-8.
43. Tan JA, Dehghan N, Chen W, Xie H, Esdaile JM, Avina-Zubieta JA. Mortality in ANCA-associated vasculitis: a meta-analysis of observational studies. Ann Rheum Dis 2017;76:1566-74.

44. Yamagata K, Usui J, Saito C, Yamaguchi N, Hirayama K, Mase K, et al. ANCA-associated systemic vasculitis in Japan: clinical features and prognostic changes. Clin Exp Nephrol 2012;16:580-8.

45. Li ZY, Gou SJ, Chen M, Zhao MH. Predictors for outcomes in patients with severe ANCA-associated glomerulonephritis who were dialysis-dependent at presentation: a study of 89 cases in a single Chinese center. Semin Arthritis Rheum 2013;42:515-21.

46. Lee SW, Yu MY, Baek SH, Ahn SY, Kim S, Na KY, et al. Long-term prognosis of anti-neutrophil cytoplasmic antibody-negative renal vasculitis: cohort study in Korea. J Korean Med Sci 2016;31:542-6.

47. Mun CH, Yoo J, Jung SM, Song JJ, Park YB, Lee SW. The initial predictors of death in 153 patients with ANCA-associated vasculitis in a single Korean centre. Clin Exp Rheumatol 2018;36 Suppl 111:65-72.

48. Holle JU, Gross WL, Latza U, Nölle B, Ambrosch P, Heller M, et al. Improved outcome in 445 patients with Wegener's granulomatosis in a German vasculitis center over four decades. Arthritis Rheum 2011;63:257-66.

49. Cartin-Ceba R, Golbin JM, Keogh KA, Peikert T, Sánchez-Menéndez M, Ytterberg SR, et al. Rituximab for remission induction and maintenance in refractory granulomatosis with polyangiitis (Wegener's): ten-year experience at a single center. Arthritis Rheum 2012;64:3770-8.

50. Bligny D, Mahr A, Toumelin PL, Mouthon L, Guillevin L. Predicting mortality in systemic Wegener's granulomatosis: a survival analysis based on 93 patients. Arthritis Rheum 2004;51:83-91.

51. Charlier C, Henegar C, Launay O, Pagnoux C, Berezné A, Bienvenu B, et al. Risk factors for major infections in Wegener granulomatosis: analysis of 113 patients. Ann Rheum Dis 2009;68:658-63.

52. Godeau B, Mainardi JL, Roudot-Thoraval F, Hachulla E, Guillevin L, Huong Du LT, et al. Factors associated with Pneumocystis carinii pneumonia in Wegener's granulomatosis. Ann Rheum Dis 1995;54:991-4.

53. Charles P, Néel A, Tieulié N, Hot A, Pugnet G, Decaux O, et al. Rituximab for induction and maintenance treatment of ANCAassociated vasculitides: a multicentre retrospective study on 80 patients. Rheumatology (Oxford) 2014;53:532-9.

54. Weidanz F, Day CJ, Hewins P, Savage CO, Harper L. Recurrences and infections during continuous immunosuppressive therapy after beginning dialysis in ANCA-associated vasculitis. Am J Kidney Dis 2007;50:36-46.

55. Palsson R, Choi HK, Niles JL. Opportunistic infections are preceded by a rapid fall in antineutrophil cytoplasmic antibody (ANCA) titer in patients with ANCA associated vasculitis. J Rheumatol 2002;29:505-10.

56. Yoo J, Jung SM, Song JJ, Park YB, Lee SW. Birmingham vasculitis activity and chest manifestation at diagnosis can predict hospitalised infection in ANCA-associated vasculitis. Clin Rheumatol 2018;37:2133-41.

57. Ni J, Qiu LJ, Hu LF, Cen H, Zhang M, Wen PF, et al. Lung, liver, prostate, bladder malignancies risk in systemic lupus erythematosus: evidence from a meta-analysis. Lupus 2014;23:284-92.

58. Liang Y, Yang Z, Qin B, Zhong R. Primary Sjogren's syndrome and malignancy risk: a systematic review and meta-analysis. Ann Rheum Dis 2014;73:1151-6.

59. Onishi A, Sugiyama D, Kumagai S, Morinobu A. Cancer incidence in systemic sclerosis: meta-analysis of population-based cohort studies. Arthritis Rheum 2013;65:1913-21.

60. Iaccarino L, Ghirardello A, Bettio S, Zen M, Gatto M, Punzi L, et al. The clinical features, diagnosis and classification of dermato- 
myositis. J Autoimmun 2014;48-49:122-7.

61. Elinav E, Nowarski R, Thaiss CA, Hu B, Jin C, Flavell RA. Inflammation-induced cancer: crosstalk between tumours, immune cells and microorganisms. Nat Rev Cancer 2013;13:759-71.

62. Mahr A, Heijl C, Le Guenno G, Faurschou M. ANCA-associated vasculitis and malignancy: current evidence for cause and consequence relationships. Best Pract Res Clin Rheumatol 2013;27:45-56.

63. Hoffman GS, Kerr GS, Leavitt RY, Hallahan CW, Lebovics RS, Travis WD, et al. Wegener granulomatosis: an analysis of 158 patients. Ann Intern Med 1992;116:488-98.

64. Knight A, Askling J, Ekbom A. Cancer incidence in a populationbased cohort of patients with Wegener's granulomatosis. Int J Cancer 2002;100:82-5.

65. Heijl C, Harper L, Flossmann O, Stücker I, Scott DG, Watts RA, et al. Incidence of malignancy in patients treated for antineutrophil cytoplasm antibody-associated vasculitis: follow-up data from European Vasculitis Study Group clinical trials. Ann Rheum Dis 2011;70:1415-21.

66. Faurschou M, Sorensen IJ, Mellemkjaer L, Loft AG, Thomsen BS, Tvede N, et al. Malignancies in Wegener's granulomatosis: incidence and relation to cyclophosphamide therapy in a cohort of 293 patients. J Rheumatol 2008;35:100-5.

67. Wester Trejo MAC, Bajema IM, van Daalen EE. Antineutrophil cytoplasmic antibody-associated vasculitis and malignancy. Curr Opin Rheumatol 2018;30:44-9.

68. Yoo J, Ahn SS, Jung SM, Song JJ, Park YB, Lee SW. Cancer development in Korean patients with ANCA-associated vasculitis: a single centre study. Clin Exp Rheumatol 2018;36 Suppl 111:73-7.

69. Honda T, Uehara T, Matsumoto G, Arai S, Sugano M. Neutrophil left shift and white blood cell count as markers of bacterial infection. Clin Chim Acta 2016;457:46-53.

70. Nigro KG, O'Riordan M, Molloy EJ, Walsh MC, Sandhaus LM. Performance of an automated immature granulocyte count as a predictor of neonatal sepsis. Am J Clin Pathol 2005;123:618-24.

71. Nahm CH, Choi JW, Lee J. Delta neutrophil index in automated immature granulocyte counts for assessing disease severity of patients with sepsis. Ann Clin Lab Sci 2008;38:241-6.

72. Yoo J, Ahn SS, Jung SM, Song JJ, Park YB, Lee SW. Delta neutrophil index is associated with vasculitis activity and risk of relapse in ANCA-associated vasculitis. Yonsei Med J 2018;59:397-405.

73. Evans TC, Jehle D. The red blood cell distribution width. J Emerg Med 1991;9 Suppl 1:71-4.

74. Tecer D, Sezgin M, Kanık A, Çncel NA, Öimen ÖB, Biçer A, et al. Can mean platelet volume and red blood cell distribution width show disease activity in rheumatoid arthritis? Biomark Med 2016; 10:967-74.

75. Aksoy ŞN, Savaş E, Sucu M, Kisacik B, Kul S, Zengin O. Association between red blood cell distribution width and disease activity in patients with Behçet's disease. J Int Med Res 2015;43:765-73.

76. Kim HJ, Yoo J, Jung SM, Song JJ, Park YB, Lee SW. Red blood cell distribution width can predict vasculitis activity and poor prognosis in ganulomatosis with polyangiitis. Yonsei Med J 2018;59: 294-302.

77. Leader A, Pereg D, Lishner M. Are platelet volume indices of clinical use? A multidisciplinary review. Ann Med 2012;44:805-16.

78. Kisacik B, Tufan A, Kalyoncu U, Karadag O, Akdogan A, Ozturk MA, et al. Mean platelet volume (MPV) as an inflammatory marker in ankylosing spondylitis and rheumatoid arthritis. Joint Bone Spine 2008;75:291-4.

79. Kim HJ, Jung SM, Song JJ, Park YB, Lee SW. Mean platelet volume can estimate the current vasculitis activity of microscopic polyangiitis. Rheumatol Int 2018;38:1095-101.

80. Kim Y, Choi H, Jung SM, Song JJ, Park YB, Lee SW. Systemic im- mune-inflammation index could estimate the cross-sectional high activity and the poor outcomes in immunosuppressive drugnaïve patients with ANCA-associated vasculitis. Nephrology (Carlton) 2018 Sep 11 [Epub]. Available at: https://doi.org/10.1111/ nep.13491.

81. Ahn SS, Jung SM, Song JJ, Park YB, Lee SW. Neutrophil to lymphocyte ratio at diagnosis can estimate vasculitis activity and poor prognosis in patients with ANCA-associated vasculitis: a retrospective study. BMC Nephrol 2018;19:187.

82. Park HJ, Jung SM, Song JJ, Park YB, Lee SW. Platelet to lymphocyte ratio is associated with the current activity of ANCA-associated vasculitis at diagnosis: a retrospective monocentric study. Rheumatol Int 2018;38:1865-71.

83. Ahn SS, Yoo J, Jung SM, Song JJ, Park YB, Lee SW. Clinical role of albumin to globulin ratio in microscopic polyangiitis: a retrospective monocentric study. Clin Rheumatol 2018 Sep 15 [Epub]. Available at: https://doi.org/10.1007/s10067-018-4292-y.

84. Moon JS, Ahn SS, Park YB, Lee SK, Lee SW. C-reactive protein to serum albumin ratio is an independent predictor of all-cause mortality in patients with ANCA-associated vasculitis. Yonsei Med J 2018;59:865-71.

85. Charles Jennette J, Xiao H, Hu P. Complement in ANCA-associated vasculitis. Semin Nephrol 2013;33:557-64.

86. Choi H, Kim Y, Jung SM, Song JJ, Park YB, Lee SW. Low serum complement 3 level is associated with severe ANCA-associated vasculitis at diagnosis. Clin Exp Nephrol 2018 Aug 23 [Epub]. Availabla at: https://doi.org/10.1007/s10157-018-1634-7.

87. Koldingsnes W, Nossent JC. Baseline features and initial treatment as predictors of remission and relapse in Wegener's granulomatosis. J Rheumatol 2003;30:80-8.

88. Ahn SS, Park ES, Jung SM, Song JJ, Park YB, Lee SW. Echocardiographic features in patients with ANCA-associated vasculitis within 3 months before and after diagnosis. Clin Rheumatol 2017;36: 2751-9.

89. Berden AE, Ferrario F, Hagen EC, Jayne DR, Jennette JC, Joh K, et al. Histopathologic classification of ANCA-associated glomerulonephritis. J Am Soc Nephrol 2010;21:1628-36.

90. Geetha D, Seo P. Renal transplantation in the ANCA-associated vasculitides. Am J Transplant 2007;7:2657-62.

91. Mukhtyar C, Flossmann O, Hellmich B, Bacon P, Cid M, CohenTervaert JW, et al. Outcomes from studies of antineutrophil cytoplasm antibody associated vasculitis: a systematic review by the European League Against Rheumatism systemic vasculitis task force. Ann Rheum Dis 2008;67:1004-10.

92. Hruskova Z, Geetha D, Tesar V. Renal transplantation in antineutrophil cytoplasmic antibody-associated vasculitis. Nephrol Dial Transplant 2015;30 Suppl 1:i159-63.

93. Schmitt WH, van der Woude FJ. Organ transplantation in the vasculitides. Curr Opin Rheumatol 2003;15:22-8.

94. Chaigne B, Guillevin L. Unsolved questions and concerns about treatment of anti-neutrophil cytoplasm antibody-associated vasculitides. Clin Exp Rheumatol 2016;34(3 Suppl 97):S121-8.

95. Park ES, Ahn SS, Jung SM, Song JJ, Park YB, Lee SW. Renal outcome of kidney-transplantation in Korean recipients with ANCA-associated vasculitis. Clin Exp Rheumatol 2018;36 Suppl 111:115-20.

96. Sun J, Zhang Y, Liu L, Liu G. Diagnostic accuracy of combined tests of anti cyclic citrullinated peptide antibody and rheumatoid factor for rheumatoid arthritis: a meta-analysis. Clin Exp Rheumatol 2014;32:11-21.

97. Mariette X. Lymphomas complicating Sjögren's syndrome and hepatitis $\mathrm{C}$ virus infection may share a common pathogenesis: chronic stimulation of rheumatoid factor B cells. Ann Rheum Dis 2001;60:1007-10. 
98. Moon JS, Lee DD, Park YB, Lee SW. Rheumatoid factor false positivity in patients with ANCA-associated vasculitis not having medical conditions producing rheumatoid factor. Clin Rheumatol 2018;37:2771-9.

99. Reininger L, Berney T, Shibata T, Spertini F, Merino R, Izui S. Cryoglobulinemia induced by a murine IgG3 rheumatoid factor: skin vasculitis and glomerulonephritis arise from distinct pathogenic mechanisms. Proc Natl Acad Sci U S A 1990;87:10038-42.

100. Ullman S, Høier-Madsen M, Halberg P, Jans H, Sylvest J. Deposits of immunoglobulins and complement in skin of patients with rheumatoid arthritis. Influence of anti-rheumatic treatment. Scand J Rheumatol 1979;8:119-23.

101. Julian BA, Czerkinsky C, Russell MW, Galla JH, Koopman WJ, Mestecky J, et al. Striking elevation of serum IgA, IgA-containing immune complexes, and IgA rheumatoid factor in clinically silent dermatitis herpetiformis. Am J Kidney Dis 1987;10:378-84.

102. Kudou M, Yasuba H, Kobayashi Y, Hamada K, Kita H. Correlation between rheumatoid factor and peripheral eosinophil count in chronic eosinophilic pneumonia. Respirology 2006;11: 830-2.
103. Yokomori H, Oda M, Kaneko F, Kawachi S, Tanabe M, Yoshimura K, et al. Lymphatic marker podoplanin/D2-40 in human advanced cirrhotic liver--re-evaluations of microlymphatic abnormalities. BMC Gastroenterol 2010;10:131.

104. Jiao J, Friedman SL, Aloman C. Hepatic fibrosis. Curr Opin Gastroenterol 2009;25:223-9.

105. De Santis M, Crotti C, Selmi C. Liver abnormalities in connective tissue diseases. Best Pract Res Clin Gastroenterol 2013;27:543-51.

106. Adler M, Gulbis B, Moreno C, Evrard S, Verset G, Golstein P, et al. The predictive value of FIB-4 versus FibroTest, APRI, FibroIndex and Forns index to noninvasively estimate fibrosis in hepatitis $\mathrm{C}$ and nonhepatitis C liver diseases. Hepatology 2008;47:762-3.

107. Lee SW, Kim DY, Ahn SH, Park YB, Han KH, Park JY. Subclinical but significant liver fibrosis in patients with ANCA-associated vasculitis. Clin Exp Rheumatol 2018 Oct 11 [In press].

108. Lee SW, Kim DY, Ahn SH, Park YB, Han KH, Park JY. HBsAgnegative and anti-HBc-positive in eosinophilic granulomatosis with polyangiitis: a retrospective pilot study. Rheumatol Int 2018; 38:1531-8 